OPEN ACCESS

Edited by:

Rita De Matteis,

Università degli Studi di Urbino Carlo

Bo, Italy

Reviewed by:

Denis Richard,

Laval University, Canada

Vicente Lahera,

Complutense University of Madrid,

Spain

*Correspondence:

Ana M. Rodríguez

amrodriguez@uib.es

Specialty section:

This article was submitted to Integrative Physiology,

a section of the journal

Frontiers in Physiology

Received: 09 November 2018

Accepted: 18 December 2018

Published: 10 January 2019

Citation:

Reynés B, Palou M, Rodríguez AM and Palou A (2019) Regulation of

Adaptive Thermogenesis and

Browning by Prebiotics and

Postbiotics. Front. Physiol. 9:1908.

doi: 10.3389/fphys.2018.01908

\section{Regulation of Adaptive Thermogenesis and Browning by Prebiotics and Postbiotics}

\author{
Bàrbara Reynés ${ }^{1,2,3}$, Mariona Palou 1,3, Ana M. Rodríguez ${ }^{1,2,3 *}$ and Andreu Palou ${ }^{1,2,3}$ \\ ${ }^{1}$ CIBER Fisiopatología de la Obesidad y Nutrición (CIBERobn), Madrid, Spain, ${ }^{2}$ Institut d'Investigació Sanitària Illes Balears \\ (IdISBa), Palma de Mallorca, Spain, ${ }^{3}$ Laboratory of Molecular Biology, Nutrition and Biotechnology, University of the Balearic \\ Islands, Palma de Mallorca, Spain
}

Prebiotics are non-digestible food components able to modify host microbiota toward a healthy profile, concomitantly conferring general beneficial health effects. Numerous research works have provided wide evidence regarding the effects of prebiotics on the protection against different detrimental phenotypes related to cancer, immunity, and features of the metabolic syndrome, among others. Nonetheless, one topic less studied so far, but relevant, relates to the connection between prebiotics and energy metabolism regulation (and the prevention or treatment of obesity), especially by means of their impact on adaptive (non-shivering) thermogenesis in brown adipose tissue (BAT) and in the browning of white adipose tissue (WAT). In the present review, a key link between prebiotics and the regulation of adaptive thermogenesis and lipid metabolism (in both BAT and WAT) is proposed, thus connecting prebiotic consumption, microbiota selection (especially gut microbiota), production of microbiota metabolites, and the regulation of energy metabolism in adipose tissue, particularly regarding the effects on browning promotion, or on BAT recruitment. In this sense, various types of prebiotics, from complex carbohydrates to phenolic compounds, have been studied regarding their microbiota-modulating role and their effects on crucial tissues for energy metabolism, including adipose tissue. Other studies have analyzed the effects of the main metabolites produced by selected microbiota on the improvement of metabolism, such as short chain fatty acids and secondary bile acids. Here, we focus on state-of-the-art evidence to demonstrate that different prebiotics can have an impact on energy metabolism and the prevention or treatment of obesity (and its associated disorders) by inducing or regulating adaptive thermogenic capacity in WAT and/or BAT, through modulation of microbiota and their derived metabolites.

Keywords: beige adipocytes, brite adipocytes, brown adipose tissue, microbiota, obesity, prebiotics, postbiotics, UCP1

\section{INTRODUCTION}

Obesity is one of the major health problems throughout the world (Bray et al., 2017). Current obesity treatment strategies, mainly based on food intake control, seem not to be enough to address this multifactorial disorder of pandemic prevalence. In this sense, studies to counteract obesity development based on energy expenditure are recently booming (Marlatt and Ravussin, 2017). In rodents, browning of white adipose tissue (WAT), and especially adaptive thermogenesis 
of brown adipose tissue (BAT), are relevant contributors to energy expenditure, being critical for energy balance and body weight maintenance. Uncoupling Protein 1 (UCP1) is the main effector of adaptive (non-shivering) thermogenesis in brown adipocytes, by uncoupling oxidative phosphorylation (Palou et al., 1998; Rodriguez and Palou, 2004). Although UCP1 is mainly expressed in BAT, browning is defined as "any significantly increased UCP1 expression at the mRNA level occurring in what are normally considered as WAT depots" (Nedergaard and Cannon, 2014). In the literature, cells expressing UCP1 in WAT can be referred to as beige or brite (brown-in-white), among other denominations (Nedergaard and Cannon, 2014). In contrast to rodents, adult humans do not exhibit well-defined BAT. However, the presence of discrete areas of active BAT-mainly in the cervical, supraclavicular, axillary, and paravertebral regions, activated in response to certain adrenergic stimuli-has been described in adult humans (Nedergaard et al., 2007). It must be noted that, unlike BAT thermogenesis, the role of beige WAT significantly influencing energy metabolism has been questioned, at least in response to chronic cold and adrenergic agonist treatment, even in the absence of functional interscapular BAT (Labbe et al., 2016, 2018). Nevertheless, although the real relevance of WAT browning to treat metabolic disorders in humans is still unclear (Bartelt and Heeren, 2014), all these findings have opened a new window to study novel treatment strategies of obesity based on enhancing energy expenditure capacity by induction of BAT recruitment and browning of WAT. Cold exposure is the main physiological thermogenic activator, although other physiological situations such as overfeeding can also physiologically activate adaptive thermogenesis (dietinduced thermogenesis) (Rothwell and Stock, 1979; Landsberg et al., 1984). In this sense, the role of the sympathetic nervous system (SNS) is crucial; SNS activation by cold exposure, as well as other thermogenic inductors which promote the release of norepinephrine, stimulates the expression and function of UCP1 in BAT and browning in WAT (Landsberg et al., 1984; Palou et al., 1998; Wu et al., 2012).

Although numerous stimuli, bioactive compounds, and physiological situations have been studied regarding the activation of adaptive thermogenesis, here we will focus on a less explored field, concerning the possible role of prebiotics and microbiota in thermogenic capacity modulation. Microbiota has emerged over the last few years as a key factor modulating different aspects of host health (VillanuevaMillan et al., 2015), and these aspects seem to include adaptive thermogenesis regulation. For instance, a study in mice showed that depletion of gut microbiota of genetically or diet induced obese mice increases browning induction in WAT (SuárezZamorano et al., 2015). Moreover, the main physiological activator of adaptive thermogenesis commented above, i.e., cold exposure, has been shown to induce adaptations in gut microbiota profile, which have been related to thermogenic and browning activation. Further, microbiota transplantation of cold exposed mice to germ free mice enhances cold tolerance, related to beige/brown fat formation in WAT (both inguinal and perigonadal) and, to a lesser extent, to BAT recruitment
(Chevalier et al., 2015), as we will discuss more extensively below.

Therefore, the relationship between the host microbiota and BAT thermogenesis or WAT browning becomes a focus of interest, even more so when taking into account the fact that the microbiota is physiologically and nutritionally mouldable. One effective physiological way to modulate microbiota, especially gut microbiota, is by the use of pro and prebiotics, especially prebiotics, which can potentially affect to large number of gut microbial species. A prebiotic is defined, at present, as "a substrate that is selectively utilized by host microorganisms conferring a health benefit," and this modern definition is broad enough to expand the concept from classical carbohydrate molecules to non-carbohydrate compounds, as well as to different applications and sites of action beyond the gastrointestinal tract (Gibson et al., 2017). Sometimes the prebiotic concept has been confused, due to partial overlap in the type of molecules involved, with the concept of "dietary fiber." Nevertheless, there are significant functional, health-related and structural differences. Dietary fiber has been defined as "nondigestable carbohydrates and lignin that are intrinsic and intact in plants" with "functional fiber" defined as "isolated, nondigestible carbohydrates that have beneficial physiological effects in humans" (Slavin, 2013). Therefore, the definition of fiber is vague in terms of functional/physiological effects and does not include other bioactive compounds distinct to carbohydrates and lignin. The prebiotic concept goes far beyond the fiber concept to include non-carbohydrate substances, applications in different body sites, and compounds from other categories apart from food (Gibson et al., 2017). Importantly, the prebiotic definition includes the requirement of microbiota involvement and selective mechanisms mediated by microbiota, also considering that the health beneficial effects must be documented in order to classify a substance as prebiotic (Gibson et al., 2017). Different mechanisms have emerged to explain the relationship of gut microbiota with health, and these include the modulation of metabolic endotoxemia (referring to lipopolysaccharideLPS-circulating levels, related to inflammation), gut-endocrine barrier, gut hormones, and peripheral tissue metabolism, among others (Cani and Delzenne, 2010; Geurts et al., 2014; Cani and Everard, 2016). Among the mechanisms involved, the metabolic effects of the products released by the microbiota after their processing, i.e., postbiotics, are gaining a lot of interest and are the focus of specific research. Postbiotics can be defined as "those molecules released by bacteria and other microorganisms that when administered in adequate amount confer health benefits to the host" (Maguire and Maguire, 2018). For instance, short chain fatty acids (SCFA) and different bile acid species are postbiotics that have gained an important focus of attention, as will be discussed below.

Taking into consideration all these antecedents, this review explores the potential link between prebiotics and the regulation of thermogenic capacity and browning, through the modulation of microbiota and postbiotics, as a tool to prevent or combat obesity and its related metabolic disorders. We propose that there is an important impact of microbiota modulation by prebiotics on the regulation of adaptive thermogenic capacity; and that 
the management of host microbiota by prebiotics might have a prominent role in the thermogenic process, with therapeutic potential.

\section{MICROBIOTA, EUBIOSIS, AND DYSBIOSIS}

The microbiota is a complex ecosystem of microorganisms, including bacteria, viruses, protozoa and fungi, which live in the human body; about $70 \%$ of the microbiota is found in the gastrointestinal tract, where 100 trillion microorganisms coexist, which amounts to more than 10 times the total human cells (Pascale et al., 2018). The most important bacteria populations are in the colon, where a certain symbiosis with the host exists, key for health maintenance (Pascale et al., 2018). Most of the gut microbiota is composed of bacteria, with some predominant phyla such as Firmicutes, Bacteroidetes, Actinobacteria, Verrucomicrobia, and Proteobacteria, and Methanobacteriales of the phylum Euryarchaeota (Diamant et al., 2011; Chakraborti, 2015). Notably, gut microbiota could be used as a microbiological "fingerprint" since, at the taxonomic level, it exhibits important differences between individuals. In this sense, host genotype, environment and diet influence gut microbiota, responsible for these individual differences (Iebba et al., 2016).

A correct symbiosis between the intestinal microbiota and the host is required for the right production of substances and metabolites necessary for host health maintenance (Boulangé et al., 2016). This correct symbiosis, and the qualitative and quantitative equilibrium between the different species present in the gut microbiota is defined as eubiosis (Iebba et al., 2016), also known as normobiosis, and is key for well-being and health (Petschow et al., 2013). On the other hand, dysbiosis is the term that defines an ecosystem where the microbiota species do not live in harmony, which negatively affects host health; it is usually associated to reduced microbial diversity and the predominance of harmful species, creating a disease-prone situation (Petschow et al., 2013; Zhang et al., 2015).

\section{MICROBIOTA AND OBESITY, AND ITS METABOLIC DISORDERS}

As suggested above, a healthy gut microbiota profile contributes to improve host metabolism, through dynamic changes in metabolites, nutrients, and vitamins, and to maintain energy homeostasis (Althani et al., 2016). Meanwhile, alterations in the microbiota profile have been related to the development of some diseases, including obesity and the metabolic disorders that accompany it. In this sense, obese people have been described to present significant differences in the composition of gut microbiota compared to lean people and, concretely, bacteria from the phylum Bacteroidetes decrease, whereas bacteria from the phylum Firmicutes, such as Bacillaceae and Clostridiaceae, increase in the obese state, especially related to prolonged exposure to a high-fat (HF) diet (Pascale et al., 2018). The detrimental change in microbiota composition may result in an augmented energy production from undigested materials, leading to a dysregulation in energy homeostasis (Pascale et al.,
2018). Moreover, it is also related to other numerous metabolic disturbances, associated with and/or contributing to obesity, such as changes in lipogenesis, triglyceride storage, hepatic steatosis, insulin sensitivity, control of food intake, etc., and the promotion of meta-inflammation (or low grade inflammation) (Cani and Delzenne, 2009). Thus, beyond microbiota involvement in energy utilization from diet, the microbiota seems to play a very relevant role in energy homeostasis, metabolism, and obesity development, thus explaining the increasing interest in analyzing the "obesity-microbiota" link in the last few years (Davis, 2016). Interestingly, differences in intestinal microbiota may even precede overweight development, a conclusion reached for the first time by Kalliomäki et al. (2008). They compared children who remained normal weight with children developing overweight/obesity at the age of 7 years, reporting that the genus Bifidobacterium was higher in number at an early age (6-12 months) (when no BMI differences were yet evident) in the posterior normal weight group compared to the overweight group (Kalliomäki et al., 2008). In addition, fecal numbers of $S$. aureus were lower in children remaining normal weight than in children developing overweight (Kalliomäki et al., 2008). These findings strongly reinforce the importance of microbiota in body weight control. In the same line, germ free C57BL/6J male mice fed a HF/high-sucrose diet are protected against dietinduced obesity in contrast to mice with normal gut microbiota (Bäckhed et al., 2007). This resistance to overweight in germ free mice was related to an increase, in liver and skeletal muscle, in the activity of AMP activated protein kinase (AMPK) and key downstream targets (related to the oxidation of fatty acids), together with the induction of the transcriptional coactivator peroxisome proliferator activated receptor gamma coactivator $1 \alpha(\mathrm{PGC} 1 \alpha)$ (another key molecular regulator of energy metabolism) (Bäckhed et al., 2007). Therefore, these findings also support the idea of a marked role of microbiota in energy balance, and provide a clue for the key molecular mechanisms involved.

Bearing in mind this connection between gut microbiota and health, it is clear that prebiotic agents, or other types of gut microbiota modulators, might be considered as agents able to prevent or improve gut dysbiosis and obesity-related metabolic disorders. For instance, Ganoderma lucidum, a traditional antidiabetic medical mushroom, reduces dysbiosis in diet-induced obese mice, related to the decrease in the Firmicutes/Bacteroidetes ratio and endotoxin (LPS) levels (Chang et al., 2015). These results were associated with decreased body weight, reduced inflammation, and improved insulin sensitivity in these animals (Chang et al., 2015). Interestingly, the beneficial effects of this prebiotic are transmissible to other diet-induced obese mice through feces transplantation. This suggests that the replacement of a microbial population by a new one, associated with the amelioration of dysbiosis, might confer beneficial health effects to the host (Chang et al., 2015). Recent results with transgenic mice with constitutive production of $\omega 3$ polyunsaturated fatty acids (PUFA) also point to the same idea. In this model, the microbiota modulation by $\omega 3$ PUFA improves the metabolic profile of high fat (HF) diet fed mice which are protected against obesity, also maintaining a normal gut barrier function and with reduced metabolic endotoxemia (Bidu et al., 2018). 
Microbiota (fecal) transplantation from the transgenic animals to metabolically altered (by an obesogenic diet) wild type animals is able to improve metabolic profile of the latter and reverse weight gain (Bidu et al., 2018); therefore, these results also suggest an important relationship between gut microbiota and protection against obesity and the related altered metabolism.

\section{IMPACT OF GUT MICROBIOTA ON BAT THERMOGENESIS AND WAT BROWNING}

Different aspects could be important to explain the relationship between gut microbiota and protection against obesity and its metabolic disorders, but when considering the main elements involved in overweight development, one outstanding factor is energy expenditure, where adaptive thermogenesis can play a crucial role. Different research works show an association between modulation of gut microbiota and thermogenic capacity in both BAT and WAT (browning). As far as we know, a link between gut microbiota and BAT metabolism was first shown by Mestdagh et al. (2012). Particularly when comparing conventional and germ-free mice, the authors showed that the absence of gut microbiota activates BAT and hepatic lipid catabolism and inhibits lipogenesis (Mestdagh et al., 2012). Following these lines, other authors have revealed relevant effects of microbiota on BAT thermogenesis and WAT browning, through cold microbiota transplantation experiments. Specifically, Chevalier et al. (2015) showed that cold exposure induces several changes in the microbiota composition and that cold microbiota transplantation (from $6^{\circ} \mathrm{C}$ exposed $\mathrm{C} 57 \mathrm{Bl} / 6$ male mice, for 25 days) to germ free mice induces the expression of the gene coding for UCP1 in BAT, together with increased resting energy expenditure. Likewise, they also revealed that this microbiota transplantation induces browning (with the appearance of UCP1 positive adipocytes) in the inguinal and perigonadal WAT depots. Further supporting the browning phenotype, the expression of some brown/brite markers (Ucp1, Cidea, and Ppargc1a) (the genes coding for UCP1, cell deathinducing DFFA-Like effector A -CIDEA- and PGC1 $\alpha$ ) was also induced (Chevalier et al., 2015). Therefore, these studies suggest that cold microbiota transplantation induces browning in WAT and, to a lesser extent, BAT recruitment, possibly related to increased energy dissipation (Chevalier et al., 2015). Similarly, Zietak et al. showed a marked change in microbiota composition in mice exposed to $12^{\circ} \mathrm{C}$ for 4 weeks, characterized by the increase and decrease in bacterial genera associated to leanness and obesity, respectively, accompanied by an increase in Ucp1 expression in BAT and WAT (Zietak et al., 2016). Moreover, they also demonstrated that fecal transplantation from cold exposed mice to germ free mice protected the latter from HF-associated disturbances after 6 weeks, increasing protein expression of UCP1 in BAT, reducing body adiposity increase, and improving insulin sensitivity, when compared to germ free mice transplanted with microbiota from $29^{\circ} \mathrm{C}$ exposed mice. In this case, in the germ free cold microbiota transplanted mice, no Ucp 1 gene expression in inguinal WAT was observed compared to the mice recipient from $29^{\circ} \mathrm{C}$ microbiota, and the brown phenotype in inguinal WAT was considered minor compared to the marked interscapular BAT thermogenic phenotype observed (Zietak et al., 2016). This was unlike the study of Chevalier et al. discussed above, where browning was considered very relevant (Chevalier et al., 2015). Another interesting question raised by the study of Zietak et al. was the possible role of the modulation of bile acid metabolism by gut microbiota (Zietak et al., 2016), thus connecting with postbiotics (bile acids can act as metabolic regulators derived from microbiota, as will be discussed below). Moreover, Zietak et al. (2017) have pointed out that the functional link between cold and gut microbiota leading to BAT increased thermogenesis is not elucidated yet. As we have previously explained, SNS is crucial for the regulation of thermogenic capacity under cold, diet and other thermogenic inductors (Landsberg et al., 1984; Palou et al., 1998; Wu et al., 2012). Nonetheless, thermogenic capacity (by BAT recruitment or WAT browning) induced by pre- or post-biotics has not necessary been linked to SNS stimulation and adrenergic signaling. In some cases (as we will see for some specific examples below), this link has been suggested, at least as part of the possible mechanistic explanation, but in other cases important evidence supports mechanisms acting by alternative pathways independent of adrenergic signaling (e.g., microbiota derived bile acids) (Zietak et al., 2017). Another condition that correlates changes in gut microbiota with WAT browning, and ameliorates insulin resistance, hepatic steatosis and obesity, is intermittent fasting (in mice), also related to the production of specific postbiotics (acetate and lactate in this case) (Li et al., 2017).

In a model of mice resistant to diet-induced obesity due to enhanced energy expenditure and BAT activity, such as is the $\beta$-Klotho KO mouse model, the relationship between microbiota and thermogenic capacity is also suggested. In this model, the usual alteration in gut microbiota profile induced by $\mathrm{HF}$ diet feeding is reduced, since $\beta$-Klotho $\mathrm{KO}$ mice fed a HF diet show a Bacteroidetes/Firmicutes ratio similar to wildtype mice fed a chow diet (Somm et al., 2017). The results reported in this work suggest that changes in secondary bile acid production are related to host thermogenesis stimulation (Somm et al., 2017), thus pointing again toward the involvement of bile acids metabolized by bacteria in the thermogenic phenotype, as will be discussed further in the section on postbiotics.

Based on the evidence given so far, it is difficult to establish how much of the energy expenditure increase observed in different models of pre and/or postbiotic supplementation can be attributed specifically to increased adaptive thermogenesis. Nevertheless, all in all, the relationship between gut microbiota and thermogenic capacity (both by BAT recruitment or WAT browning) is evident. Hence, the possibility of modulating microbiota composition by nutritional factors, in this case prebiotics, arises as an interesting physiological way of influencing energy metabolism, concretely adaptive thermogenesis, and prevent or even treat overweight/obesity and the associated metabolic disturbances, as is discussed in the next section. 


\section{IMPACT OF PREBIOTICS ON BAT THERMOGENESIS AND WAT BROWNING}

The concept of prebiotics and the importance of their health promoting effects have already been defined. There are multiple natural sources of prebiotics, such as beans/legumes, starchy fruits, cereals, onions, soybean, etc., and generally multiple kinds of vegetables, as well as other food products such as milk and fungi (Geurts et al., 2014; George Kerry et al., 2018). Moreover, many substances could be considered possible prebiotics, such as galactooligosacaccharides, inulin-type fructans, arabionoxylan, and arabinoxylan oligosaccharides, chitin-glucans from fungi or even several phenolic compounds, given that they favor the growth of some intestinal microorganisms and show potential health effects (Geurts et al., 2014; Markowiak and Slizewska, 2018).

There is extensive literature relating the consumption of diets rich in prebiotics to the improvement of food intake control, and the reduction of body fat content and body weight gain, even in overweight and obese subjects (Roberfroid et al., 2010; Requena et al., 2018). Now, with the present knowledge concerning multiple prebiotics and their effects on gut microbiota, we are able to connect prebiotics with the proposed health effects by the modulation of microbiota, postbiotics, and even deciphering the main mechanisms involved, including the induction of an increased thermogenic phenotype. Therefore, some representative prebiotics able to impact thermogenic capacity will be discussed in this section.

\section{Phenolic Compounds, Energy Metabolism, and Thermogenesis}

Phenolic compounds, or polyphenols, are secondary metabolites naturally produced by plants, which can be classified into two main groups: flavonoids and non-flavonoids. Phenolic compounds are generally characterized by the presence of at least one aromatic ring with one or more hydroxyl groups attached (Crozier et al., 2009). Polyphenols or polyphenol-rich fonts have been described to impact the relative abundance of different bacteria within the gut microbiota in various in vitro studies and animal models, by decreasing potential pathogens, such as C. perfringens and C. histolyticum, and certain Gram-negative Bacteroides spp., and inducing beneficial Clostridia, Bifidobacteria and Lactobacilli (Dueñas et al., 2015).

The impact of dietary polyphenols on obesity has also been a topic of interest. Over the years, human and animal studies have revealed that resveratrol, a polyphenol with prebiotic properties, exerts anti-obesity effects (Wang et al., 2015). There are several biological processes involved in the fat-lowering effects of resveratrol, even though the molecular mechanisms implicated have not been fully elucidated (Wang et al., 2015). An important number of studies puts the focus on in vitro effects (in cell culture models) of resveratrol, but these types of studies are not the focus of our review. Regarding in vivo studies, Alberdi et al. described that resveratrol supplementation induces the expression of some genes involved in BAT activation (cytochrome-C-oxidase subunit-2-Mtco2-, Ppargcla, sirtuin 1Sirt1-, and mitochondrial transcription factor A-Tfam), together with protein expression of UCP1, in Sprague-Daley rats fed with an obesogenic diet for 6 weeks (Alberdi et al., 2013). Besides, the combination of resveratrol with another polyphenol, quercetin, also induces UCP1 in BAT (Arias et al., 2017). One possible molecular mechanism involved in BAT activation by resveratrol supplementation is based on AMPK activation (Wang et al., 2017). Nevertheless, part of these conclusions come from the combination of in vivo and in vitro studies and it is difficult to separate the possible direct effects of resveratrol from its putative effects as a prebiotic agent. In the same line, related to the activation of thermogenesis in BAT, dietary supplementation with resveratrol and quercetin also resulted in the stimulation of browning in WAT (Arias et al., 2017). Namely, the authors showed that resveratrol and quercetin supplementation for 6 weeks in Wistar rats fed with an obesogenic diet induced the appearance of brown-like adipocytes in perirenal WAT (Arias et al., 2017). These studies highlight the link between resveratrol, with its fat lowering effects, and browning and thermogenesis activation. Other interesting study with resveratrol supplementation and WAT browning was performed with oral supplementation of newborn mice throughout lactation, which is a critical period for metabolic programming and the shaping of gut microbiota (Pico and Palou, 2013; Milani et al., 2017). In such study, Serrano et al. (2018) supplemented mice with resveratrol (or vehicle) from day 2 to 20 of life and, in the adulthood (day 90) half of the animals were subjected to an HF diet for 10 weeks. The neonatally male treated animals did no show induction of thermogenic markers in BAT (where expression of Ucp1 was decreased), but they showed resistance to weight gain and a metabolic programming toward browning in subcutaneous WAT (Serrano et al., 2018). Regarding the issue of the possible effects of resveratrol acting as a prebiotic, it must be highlighted that the direct action of resveratrol modulating gut microbiota has been demonstrated. For example, resveratrol supplementation has been associated with the attenuation of the Firmicutes/Bacteroidetes ratio and the selective growth of potential beneficial genera such as Lactobacillus and Bifidobacterium, either alone or in combination with quercetin (Larrosa et al., 2009; Etxeberria et al., 2015). It has also been reported to increase hepatic bile acid neosynthesis via gut microbiota remodeling, associated to the reduction of trimethylamine-N-oxide (TMAO) levels and atherosclerosis (Chen et al., 2016).

Other interesting polyphenols with prebiotic properties are anthocyanins. Positive effects against obesity and related alterations have been attributed to anthocyanin intake (from blueberries) (Meydani and Hasan, 2010). Anthocyanins are a major type of polyphenols, and have prebiotic properties since their low bioavailability make them likely to be metabolized by gut microbiota, and anthocyanins and their metabolites may exert a positive modulation on the intestinal bacterial population (Hidalgo et al., 2012). Relevant to this review is the described association between anthocyanins and BAT 
activation. In this sense, animal studies on supplementation with anthocyanins, or anthocyanin metabolites, have shown a relationship with thermogenic capacity of BAT and browning of WAT. More specifically, supplementation with cyanidin3-glucoside (C3G) of genetically obese mice (C57BLKS/JLeprdb/Leprdb) for 16 weeks resulted in reduction of weight gain, ameliorated glucose homeostasis impairment and hepatic steatosis, improved tolerance to cold exposure, and stimulated BAT activity and browning in subcutaneous WAT (You et al., 2017). Similarly, wild type C57BL/6J mice supplemented with C3G for 15 weeks were resistant to HF/high fructose diet induced obesity, associated with increased thermogenic capacity of BAT and browning of inguinal WAT, by increasing mitochondrial biogenesis and function (You et al., 2018). Altogether, these data reveal that $\mathrm{C} 3 \mathrm{G}$ intake may be considered as a novel approach to prevent and treat obesity.

Other polyphenols are also gaining significance in relation to their anti-obesity effects due to their thermogenic effect. For instance, supplementation with dietary green tea extract (GTE) from leaves for 8 weeks to mice fed a HF diet significantly reduces HF-induced adiposity in both WAT and BAT, by reducing both adipocyte size in WAT and lipid droplet size in BAT. It is noteworthy that markers of browning were induced in WAT in GTE treated mice, whereas markers of whitening were reduced in the BAT of these animals, suggesting a role of browning and thermogenesis induction in the anti-obesity beneficial effects of GTE intake (Neyrinck et al., 2017). Another extract rich in polyphenols used in research is camu camu (Myrciaria dubia) (an Amazonian fruit) crude extract (CC). Daily treatment with CC in mice fed a HF diet for 8 weeks markedly reshapes gut microbiota and confers protection against obesity development by increasing energy expenditure, accompanied by Ucp 1 induction in BAT and WAT, together with other markers of browning (Anhê et al., 2018). Moreover, CC-treated mice showed altered levels and composition of circulating bile acids and changes in bile acid composition (with higher unconjugated secondary bile acids) (Anhê et al., 2018). Finally, transplantation of microbiota from CC treated mice to germ-free mice partially reproduced the beneficial effects of CC supplementation (Anhê et al., 2018). One more example is capsaicin, which has also shown prebiotic properties (Kang et al., 2017). Capsaicin supplementation to male Wistar rats results in the appearance of multilocular adipocytes positive for UCP1 and CIDEA in retroperitoneal WAT, and increased expression of browning markers in inguinal WAT (Mosqueda-Solis et al., 2018).

\section{Prebiotics of Carbohydrate Nature and Thermogenesis}

Different types of carbohydrates are classified as prebiotics, and we focus on different examples with experimental data supporting their role in the regulation of adaptive thermogenesis.

In the group of non-digestible complex carbohydrates with prebiotic properties, pectins are an interesting example and, concretely, high-esterified pectin (HEP). Regarding structure, pectin is a complex heteropolysaccharide, made up of a series of covalently linked polymers, and the degree of methylation determines its usage by bacteria (Hamaker and Tuncil, 2014; Fak et al., 2015), with HEP importantly being fermented by gut bacteria. HEP is a main component of soluble dietary fiber in vegetables and fruits, and is recognized as a prebiotic (Lattimer and Haub, 2010; Hamaker and Tuncil, 2014), whose intake is associated with health-promoting effects on body weight, lipid metabolism, glucose homeostasis, etc. (Sanchez et al., 2008; Adam et al., 2015; Palou et al., 2015). Concerning the effect of HEP supplementation on BAT thermogenesis and WAT browning, we have developed studies in rats showing that chronic HEP supplementation (from apples) decreases energy efficiency and protects from fat accumulation and metabolic disturbances caused by maternal malnutrition, in part associated to increased thermogenic capacity in BAT and WAT, including the induction of UCP1 (unpublished manuscript in preparation). Therefore, HEP is a cheap, easy-to-get prebiotic with important effects on metabolic health, influencing thermogenic capacity. There are more examples of fermentable polysaccharides that have been related to adaptive thermogenesis modulation. Weitkunat et al. investigated how inulin and guar gum supplementation for 30 weeks affects the metabolic syndrome-related disorders associated to HF diet feeding in mice. The results showed that both inulin and guar gum affect gut microbiota composition but only inulin, and not guar gum supplementation, attenuates the HF diet induced body weight and fat gain, and significantly induces brown/brite markers in subcutaneous WAT (Weitkunat et al., 2017). In the same work, the authors also investigated the effect of SCFA, as will be discussed below (see section on postbiotics).

Another example of non-digestible, but in this case not complex, carbohydrate with beneficial physiological effects on metabolic health is epilactose (Murakami et al., 2015). Epilactose is a rare disaccharide proposed to prevent diet-induced obesity from studies in mice. Murakami et al. showed how HF fed mice supplemented with epilactose were protected against body and fat gain, and revealed greater Ucp1 gene expression in BAT and, surprisingly, also in muscle; they also reported decreased gene expression of macrophage infiltration markers in epididymal WAT. Moreover, supplemented mice showed a different caecum SCFA profile, with increased acetate and propionate levels (Murakami et al., 2015).

Given the above evidence, it seems clear that different types of prebiotics can affect adaptive thermogenic capacity, although this is a relative by new field where more studies are needed. Furthermore, the underlying mechanisms explaining the relationship between prebiotic supplementation and the induction of thermogenesis and browning is still not fully clear, although some works are starting to focus on unraveling such mechanisms. Although not the only one, an important point of connection between thermogenic capacity and prebiotics, as we have already suggested here, is the production of secondary metabolites by resident bacteria after fermentation of prebiotics. For instance, as already pointed out, SCFA profile and secondary bile acids might be important metabolites derived from bacteria metabolism able to connect prebiotic supplementation to thermogenic capacity regulation in BAT and WAT. Although the importance of postbiotics has been 
relatively overlooked, scientific evidence of their beneficial health effects by BAT thermogenic activation and WAT browning is progressively increasing, as will be discussed in the next section.

\section{IMPACT OF POSTBIOTICS ON BAT THERMOGENESIS AND WAT BROWNING}

Postbiotics (also known as metabiotics, biogenics, or metabolite/cell-free supernatants) are "soluble factors (products or metabolic byproducts) secreted by live bacteria or released after bacterial lysis" and they provide physiological benefits to the host (Aguilar-Toalá et al., 2018). There are multiple types of postbiotics with varied structures, such as SCFA, peptides, enzymes, teichoic acids, exo- and endo-polysaccharides, vitamins, etc. (Aguilar-Toalá et al., 2018). As key examples, here we discuss the scientific evidence of the link between BAT activation and WAT browning with bile acids and SCFA.

\section{Bile Acids and BAT Thermogenesis}

Bile acids (BA) are synthesized in liver from cholesterol, as primary bile acids, which are cholic acid $(3 \alpha, 7 \alpha, 12 \alpha$-trihydroxy$5 \beta$-cholanoic acid, CA) and chenodeoxycholic acid $(3 \alpha, 7 \alpha-$ dihydroxy-5 $\beta$-cholanoic acid, CDCA) in humans, while in rodents CDCA is metabolized to muricholic acids (less toxic) (Martinot et al., 2017). The synthesized primary BA are conjugated with the aminoacids glycine or taurine (forming bile salts), prior to their secretion, which is fundamental to their role of emulsification of lipids during digestion (Martinot et al., 2017). There are also secondary BA, which are produced by the metabolization of primary BA by gut microbiota; the most common secondary BA species are deoxycholic acid (DCA) and lithocholic acid (LCA), coming from CA and CDCA, respectively, although dozens of secondary BA are produced by the gut flora, increasing the diversity of possible secondary BA (Martinot et al., 2017). It should be taken into account that the profile of secondary bile acids might be modulated by environmental conditions which can affect gut microbiota composition. In this sense, in the work already commented above by Zietak et al. (2016), it is reported that cold exposure and diet are able to modify BA metabolism. For instance, cold acclimation at $12^{\circ} \mathrm{C}$ of C57BL6/J mice, after only 1 day, increases conjugated BA levels, related to reduced abundance of Lactobacillus, which possesses greater bile acid deconjugation capacity than other species (Zietak et al., 2016). Moreover, in this work, cold acclimation increased both the production of BA species and the expression of genes related to BA synthesis (Zietak et al., 2016).

BA regulate numerous metabolic pathways in the host via the nuclear receptor farnesoid X receptor (FXR) and the G-coupled receptor TGR5 (Martinot et al., 2017). Among these metabolic pathways, the regulation of thermogenesis is included. Watanabe et al. showed that TGR5 activation by BA promotes intracellular thyroid hormone activity and increases thermogenesis in BAT (Watanabe et al., 2006). Diet supplementation with CA to $\mathrm{HF}$ diet fed C57BL/6J mice prevents, and even reverts, diet induced obesity, related to increased energy expenditure and the induction of TGR5 receptor, which increases cAMP production, involved in increased expression of genes coding for proteins related to BAT activation (PGC1 $\alpha$ and $\beta, \mathrm{UCP} 1$ and 3, 2 iodothyronine deiodinase-D2-, straightchain acyl-CoA oxidase 1-ACO-and muscle-type carnitine palmitoyltransferase $1-\mathrm{mCPT} 1)$, therefore showing activation of thermogenesis in BAT (Watanabe et al., 2006). In this work, apart from primary BA, the authors showed, in vitro, the capacity of secondary BA (DCA) and taurine conjugates (of CA and DCA) to induce cAMP production in cells (related to TGR5 activation) (Watanabe et al., 2006). Therefore, this work demonstrates the capacity of both primary and bacteria-metabolized BA to potentially induce thermogenic activity. In another related paper, it was suggested that UCP1 expression is required for weightreducing action of bile acids (Zietak and Kozak, 2016). Other studies relate BA to BAT activation (even in humans) (e.g., Teodoro et al., 2014; Broeders et al., 2015) although focused on primary BA. Since we are focusing on postbiotics, another interesting study, which we have formerly discussed, is the work by Somm et al. with $\beta$-Klotho KO mice. Here, the authors show that $\beta$-Klotho $\mathrm{KO}$ mice have a high production of $\mathrm{CA}$ in liver, resulting in an important excess of DCA (secondary $\mathrm{BA})$ production by microbiota, suggesting that resistance of these KO mice to diet-induced obesity is mainly caused by the high production of DCA and its signaling through the receptor TGR5 (Somm et al., 2017). It is important to note that DCA is suggested to be especially responsible of the activation of TGR5 to stimulate BAT thermogenesis (Somm et al., 2017), related to its greater potency (compared with other BA) to activate TGR5 in vitro (Maruyama et al., 2002) and that, in fact, TGR5 is mainly considered as a receptor for secondary BA (Kawamata et al., 2003).

\section{Role of SCFA in BAT Thermogenesis and WAT Browning}

SCFA are important metabolites produced by gut microbiota by the fermentation of non-digestible carbohydrates. SCFAs are monocarboxylic acids of 2-6 carbons and the main SCFA for their abundance in plasma and caecum are (in order from more to less abundant) acetate, propionate, and butyrate, as measured in humans and rats (Topping and Clifton, 2001; Jakobsdottir et al., 2013; Schonfeld and Wojtczak, 2016). It should be taken into account that the microbiota profile conditions the relative proportions of individual SCFA produced; namely this, Bacteroidetes mainly produce acetate and propionate, and Firmicutes mainly butyrate (Pascale et al., 2018). However, the relationship may not be that simple and different phyla or genera are not exclusively associated to the production of a specific SCFA, e.g., a positive correlation between relative abundance of Firmicutes and plasma acetate levels in humans has been shown (Moreno-Navarrete et al., 2018).

SCFA affect a variety of biological processes in multiple organs and tissues and have been described to play a role in different aspects of energy metabolism and body weight control. For instance, diet supplementation for 4 weeks with salts of 
TABLE 1 | Studies evaluated in this review of effects of prebiotics on adaptive thermogenesis by BAT recruitment and/or WAT browning.

\begin{tabular}{llll}
\hline $\begin{array}{l}\text { Type of } \\
\text { Prebiotic }\end{array}$ & Prebiotic & Experimental design & $\begin{array}{l}\text { Main results on BAT thermogenesis } \\
\text { and WAT browning }\end{array}$ \\
\hline
\end{tabular}

\begin{tabular}{|c|c|c|}
\hline $\begin{array}{l}\text { Phenolic } \\
\text { compounds }\end{array}$ & Resveratrol & $\begin{array}{l}\mathrm{HF} / \text { high sucrose fed rats treated with } \\
\text { resveratrol (15 mg/kg body/day), quercetin } \\
\text { (30 mg/kg body/day) or both, for } 6 \text { weeks }\end{array}$ \\
\hline
\end{tabular}
Resveratrol and quercitin treatment: Appearance UCP1+multilocular cells in perirenal WAT $\uparrow$ Expression of browning markers in perirenal WAT $\uparrow$ Expression thermogenic markers Cox-2 and UCP1 in BAT
Resveratrol treatment: $\uparrow$ Expression thermogenic markers Cidea and UCP1 in BAT
个Expression thermogenic markers in BAT, including Ucp1

HF/high sucrose fed Sprague-Daley rats treated with resveratrol $(30 \mathrm{mg} / \mathrm{kg}$ body/day), for 6 weeks

NMRI mice supplemented with resveratrol (2 mg/Kg body/day) during lactation (day 2-20 of life). HF diet from day 90 of life (for 10 weeks) vs. normal fat diet

Anthocyanins

Obese male C57BLKS/J-Leprdb/Leprdb mice treated with Cyanidin 3-glucoside $(1 \mathrm{mg} / \mathrm{ml})$ for 16 weeks

HF/high fructose fed male C57BL/6J mice treated with Cyanidin 3-glucoside $(1 \mathrm{mg} / \mathrm{ml})$ for 15 weeks

Green Tea extracts

Male C57BL/6J mice fed with HF diet supplemented with green tea extract ( $0.5 \%$ green tea leaf extract) for 8 weeks

Camu Camu (Myrciaria dubia)

Male C57BL/6J mice fed with HF/high sucrose diet supplemented with Camu Camu crude extract $(200$ mg/Kg) compared to Vitamin C $(6.6 \mathrm{mg} / \mathrm{Kg})$ for 8 weeks

Capsaicin

Male Wistar rats fed with HF/high sucrose and supplemented with Capsaicin (4 $\mathrm{mg} / \mathrm{kg}$ body/day) for 8 weeks

Carbohydrates Epilactose

Inulin and guar gum
Male C57/BL6 mice fed with HF diet, supplemented with Epilactore (10\% by weight) for 8 weeks

Male C57BL/6JRj mice fed with HF diet supplemented with inulin or guar gum (7\%) for 30 weeks $\uparrow$ Expression of browning markers in inguinal WAT in males

$\uparrow$ Multilocular UCP1+ adipocytes in inguinal WAT under normal fat diet

$\downarrow$ Ucp1 expression in BAT

$\uparrow$ Energy expenditure

$\uparrow$ Expression of browning markers in

sucbutaneous WAT

个Expression of BAT thermogenic

markers and BAT activity

$\uparrow$ Energy expenditure

Better performance maintaining body

temperature compared with died induced obese mice

$\uparrow$ Mitochondrial biogenesis and function

$\uparrow$ Expression of browning markers in

inguinal WAT

个Expression thermogenic markers in BAT

$\uparrow$ Expression of browning markers in

subcutaneous WAT

$\downarrow$ Whitening in BAT

$\uparrow$ Appearance of UCP1+ adipocytes in

subcutaneous WAT depot

$\uparrow$ Energy expenditure

$\uparrow$ Interscapular temperature (tendency)

$\uparrow$ Expression of browning markers in

inguinal WAT

$\uparrow$ Expression thermogenic markers in BAT

$\uparrow A p p e a r a n c e$ of UCP1+ and CIDEA+

adipocytes in retroperitoneal WAT

$\uparrow$ Expression of browning markers in

inguinal WAT

个UCP1 expression in BAT and muscle

Inulin but not guar gum:

$\uparrow$ Body temperature

$\uparrow$ Expression of browning markers in

subcutaneous WAT
Murakami et al., 2015

Arias et al., 2017

Alberdi et al., 2013

Serrano et al., 2018

You et al., 2017

You et al., 2018

Neyrinck et al., 2017

Anhê et al., 2018

Mosqueda-Solis et al., 2018

Weitkunat et al., 2017

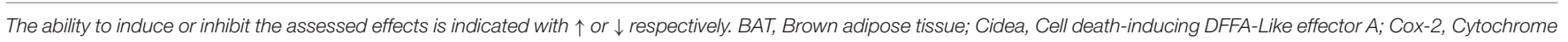
c oxidase subunit II; HF, High fat; UCP1, Uncoupling Protein 1; WAT, White adipose tissue.

butyrate $(5 \%)$, propionate $(4.3 \%)$, or acetate $(3.7 \%)$ has been described to protect against diet-induced obesity and insulin resistance in mice fed a HF diet (Lin et al., 2012). Butyrate and propionate, but not acetate, were able to stimulate gut hormones and reduce food intake (Lin et al., 2012). Propionate has also been described to inhibit lipogenesis via fatty acid synthase down-regulation in the liver, while acetate can act as a lipogenic substrate, therefore the acetate/propionate ratio 
TABLE 2 | Studies evaluated in this review of effects of postbiotics on adaptive thermogenesis by BAT recruitment and/or WAT browning.

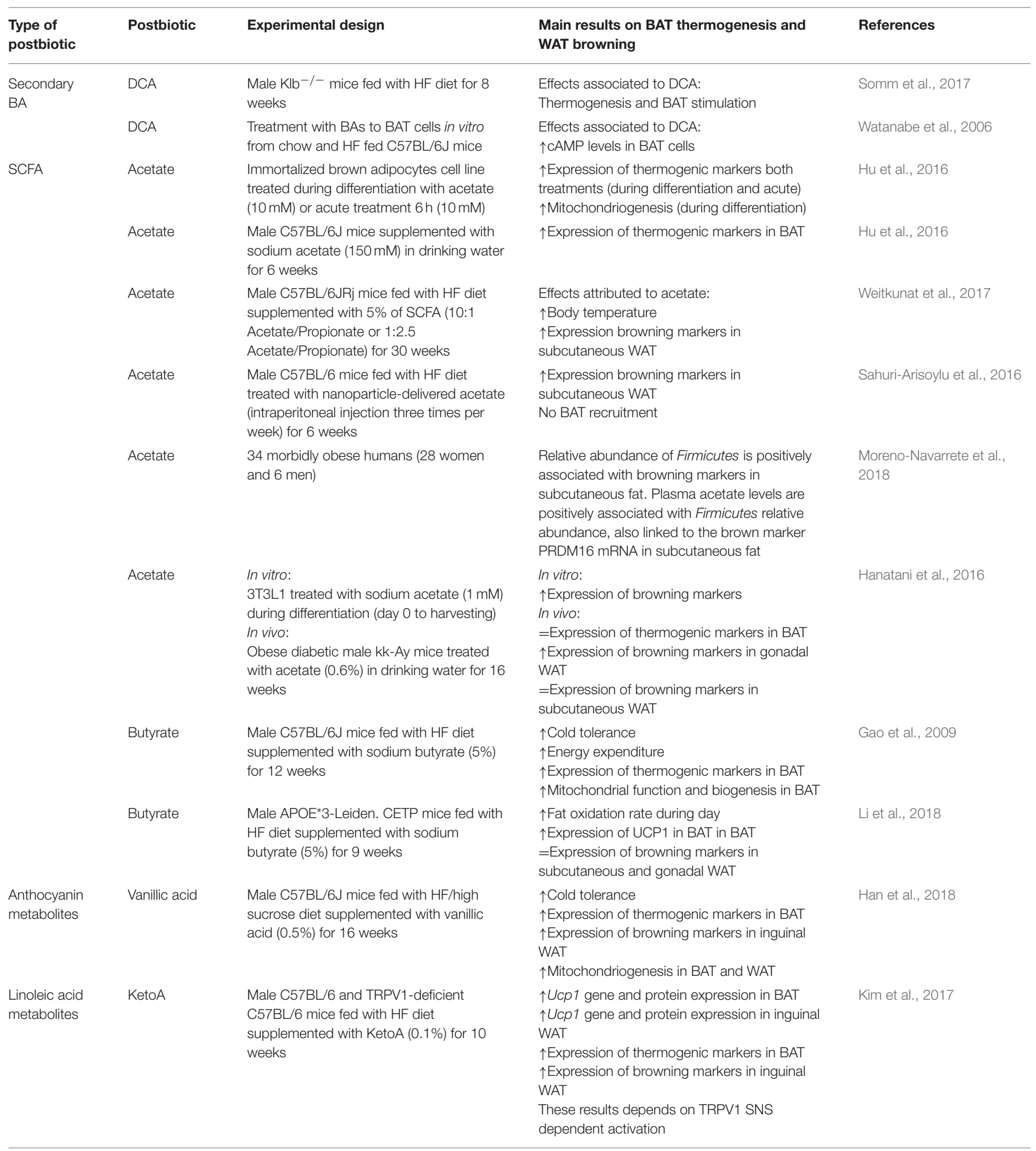

The ability to induce the assessed effects is indicated with $\uparrow$ while = indicates no significant changes. BA, Bile acids; BAT, Brown adipose tissue; DCA, Deoxycholic acid; HF, High fat; KetoA, 10-oxo-12(Z)-octadecenoic acid; PRDM16, PR domain containing 16; SCFA, Short chain fatty acids; SNS, sympathetic nervous system; TRPV1, Transient receptor potential vanilloid 1; UCP1, Uncoupling Protein 1; WAT, White adipose tissue.

seems important for de novo lipogenesis (Conterno et al., 2011). Moreover, De Vadder et al. showed that propionate and butyrate induce the expression of gluconeogenesis related genes and activate intestinal gluconeogenesis through different mechanisms, and this has metabolic benefits such as reduced body weight and adiposity, and improvement in glucose 
homeostasis, as studied in rodents (De Vadder et al., 2014). In vitro experiments revealed that butyrate can directly activate the expression of intestinal gluconeogenesis related genes (coding for glucose-6-phosphatase-G6PC-and the cytosolic form of phosphoenolpyruvate carbocykinase-PCK1), while propionate requires the gut-brain circuit involving the free fatty acid receptor 3 (FFAR3) in mice to induce intestinal gluconeogenesis (De Vadder et al., 2014). Regarding acetate, the literature points toward a complex role. As has already been commented, acetate can act as a substrate for lipogenesis, but it has also been described to activate AMPK in vitro and to reduce the expression of key genes for gluconeogenesis and lipogenesis in liver of KK$\mathrm{A}(\mathrm{y})$ diabetic mice supplemented with acetic acid, associated to reduced hyperglycaemia (Sakakibara et al., 2006). Strikingly, in the literature, there are both studies suggesting that acetate treatment induces an increase in lipogenic and adipogenic capacity (Hong et al., 2005; Ge et al., 2008; Aberdein et al., 2014) or the promotion of obesity through a gut-brain- $\beta$-cell axis (Perry et al., 2016; Trent and Blaser, 2016), and studies that suggest beneficial and protection-against-obesity effects, such as the one commented above and some others (e.g., see Kondo et al., 2009; Aoki et al., 2017, and see below). Therefore, the role of acetate is somewhat controversial. Regarding BAT thermogenesis and WAT browning, recent literature points to a role of some SCFA, as discussed below.

Concerning acetate and thermogenesis, new findings agree with the potential capacity of acetate to increase the activity of BAT or WAT browning in different models. In this sense, Hu et al. (2016) studied BAT of mice treated with sodium acetate $(150 \mathrm{mM})$ in drinking water for 6 weeks, reporting an induction of the expression of genes coding for PGC1 $\alpha$ and UCP1 as compared to controls, accompanied by morphological changes, including mitochondrial biogenesis. Moreover, treatment with acetate of immortalized brown adipocyte cells (IM-BAT), obtained by primary cell culture of BAT from C57BL/6 mice, induced adipogenesis and mitochondriogenesis, together with up-regulation of gene expression of key thermogenic markers, such as adipocyte protein 2 (AP2), PGC1 $\alpha$, and UCP1, depending on the activation of the free fatty acid receptor G proteincoupled receptor 43 (GPCR43) (FFAR2) (Hu et al., 2016). Moreover, the work of Weitkunat et al. (2017) has already been commented in the section on prebiotics of carbohydrate nature and thermogenesis; herein, the authors suggest an induction of beige markers (browning) in WAT driven by acetate. Other studies also suggest an involvement of acetate in WAT browning (Sahuri-Arisoylu et al., 2016; Moreno-Navarrete et al., 2018). Intraperitoneal injection of nanoparticules of acetate increases gene expression of brown adipocyte markers (UCP1 and PR domain containing 16-PRDM16) in subcutaneous WAT of HF diet fed mice, which might be partly responsible for the reduced obesogenic phenotype of these treated animals (SahuriArisoylu et al., 2016). However, no BAT recruitment due to acetate treatment was found in this work (Sahuri-Arisoylu et al., 2016). On the other hand, a recent study in obese humans has shown a positive significant association between brown markers PRDM16, UCP1 and thyroxine deiodinase 2-DIO2-in subcutaneous WAT and the Firmicutes relative abundance, which seems to contribute significantly to the variance of these markers in this adipose depot (MorenoNavarrete et al., 2018). Moreover, Firmicutes relative abundance associates positively with circulating acetate levels (MorenoNavarrete et al., 2018). Therefore, the authors conclude that microbiota composition influences WAT browning in these obese subjects, probably through plasma acetate (MorenoNavarrete et al., 2018). There is also in vitro evidence linking acetate with browning. For instance, 3T3-L1 adipocytes have been described to increase the expression levels of several browning markers after acetate treatment (UCP1, PRDM16, DIO2, CIDEA, and transmembrane protein 26-TMEM26-, among others) (Hanatani et al., 2016). The authors also provide in vivo evidence in mice and conclude that acetate might exert its anti-obesity effects through the dissipation of energy excess, at least in part (Hanatani et al., 2016). We have also performed in vitro experiments with murine primary cultures of adipocytes treated with acetate, showing that it induces thermogenic capacity in brown adipocytes and in recruited beige/brite adipocytes form subcutaneous WAT (unpublished manuscript in preparation). Therefore, different studies suggest that acetate might have an anti-obesity effect partly related to the induction of WAT browning, together with the recruitment of BAT, thus increasing energy expenditure capacity.

There is less evidence regarding adaptive thermogenesis capacity and other SCFA. Nevertheless, there is some evidence for butyrate as a thermogenic molecule. For instance, Gao et al. showed, among other effects, that dietary butyrate treatment in HF diet fed mice for 12 weeks induces the expression of UCP1 and PGC1 $\alpha$ in BAT and mitochondrial function and biogenesis in BAT and muscle, accompanied by an improvement in insulin sensitivity (Gao et al., 2009). Besides, Li and collaborators demonstrated in mice that chronic butyrate supplementation prevents diet-induced obesity, hypertriglyceridemia, hepatic steatosis, and hyperinsulinemia, although mainly attributed to reduction of food intake ( $\mathrm{Li}$ et al., 2018). Nevertheless, the authors also showed an activation of BAT, associated to increased fatty acid utilization from plasma triglycerides (Li et al., 2018). Moreover, it must be highlighted that butyrate treatment in mice has also been shown to promote BAT thermogenic capacity by activating UCP1 protein expression (and PCG1 $\alpha$ ) (Gao et al., 2009). In the work of Li et al., no evidence of browning in subcutaneous fat was found, suggesting the butyrate is not involved in browning induction (Li et al., 2018). Regarding propionate, indirect evidence for its involvement on adaptive thermogenic capacity is given by the fact that it can directly induce SNS activity via GPR41 (FFAR3) at sympathetic ganglia, correlated with energy consumption (Kimura et al., 2011). Moreover, in this work, GPR41 ablation (Gpr41-/mice) was associated with lower Ucp1 expression in BAT. The authors suggest an important role of SCFA-GPR41 interactions regulating SNS as a mechanism accounting for the effects of diet and pre/probiotics on body homeostasis (Kimura et al., 2011).

Overall, despite some controversial results, SCFA, particularly acetate, seem to be proper candidates to continue to be tested 


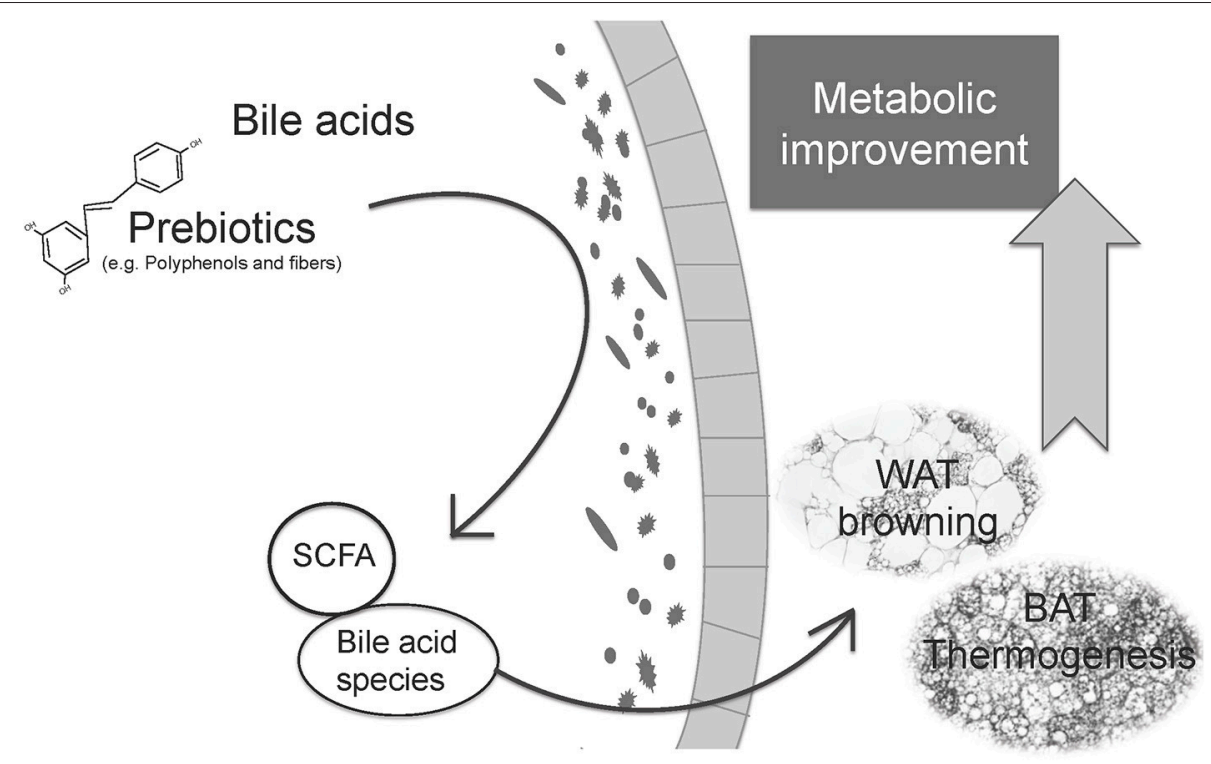

FIGURE 1 | Relationship between prebiotics, microbiota modulation and postbiotics with thermogenic capacity regulation and the improvement of metabolic health. Prebiotics can modulate gut microbiota to promote eubiosis and the selective growth of beneficial species. Microbiota can metabolize prebiotics and primary bile acids to produce specific postbiotics, some of them with proven health benefits. The microbiota changes and the postbiotics produced can influence adaptive thermogenesis, promoting BAT recruitment, and WAT browning which, in turn, boost energy expenditure and contribute to the general improvement of metabolism, with associated health benefits.

as inductors of thermogenesis in BAT and browning in WAT, in order to reduce the detrimental health effects associated to diet-induced obesity. In this sense, human studies are essential to establish a strong association between SCFA and their beneficial effects at different levels.

\section{Role of Other Postbiotics on BAT Thermogenesis and WAT Browning}

Apart from the postbiotics shown here, there are other various possibilities. For instance, we commented above that anthocyanins may have positive beneficial effects as anti-obesity factors and as thermogenesis and browning inductors (You et al., 2015, 2017) yet, interestingly, the metabolites produced by the utilization of anthocyanins by gut microbiota may also develop a role in host thermogenesis induction, similar to other postbiotics. In this sense, vanillic acid has been recently described to exhibit thermogenesis induction capacity (Han et al., 2018). Vanillic acid is one of the metabolites produced through the metabolization of anthocyanins by intestinal microbes (Keppler and Humpf, 2005). It has been reported that supplementation with vainillic acid for 16 weeks protects mice from $\mathrm{HF} / \mathrm{high}$ sucrose diet induced obesity, by activating thermogenesis in BAT and browning in WAT (Han et al., 2018). These results, again, point out the capacity of postbiotics as BAT activators, and browning agents, in the treatment or prevention of dietinduced obesity. Another interesting postbiotic is 10-oxo-12(Z)octadecenoic acid (KetoA). KetoA is a metabolite of linoleic acid produced by lactic acid bacteria in the gut able to stimulate SNS through activation of the ion channel transient receptor potential vanilloid 1 (TRPV1) in the gastrointestinal tract, concomitantly enhancing energy expenditure, related to activation of BAT function and inguinal WAT browning (Kim et al., 2017).

\section{SUMMARY AND PERSPECTIVES}

The main bibliography and evidence of the different results shown in this review, supporting the role of prebiotics and postbiotics in adaptive thermogenesis regulation, is summarized in Tables 1, 2. Additionally, Figure 1 summarizes the central idea shown in this review, linking prebiotics, microbiota modulation, and postbiotics with BAT thermogenesis and WAT browning and the improvement of metabolism.

The incipient growth of studies suggesting a connection between microbiota and energy expenditure brings to light the potential of establishing new strategies against the pandemic of obesity. It is important to highlight the opportunity of the present review, since in the last 2-3 years numerous articles have been published studying the role of microbiota and the effects of prebiotics and postbiotics on the development of obesity and, more specifically, relating them to the activation of thermogenesis in BAT and browning in WAT. Microbiota suffers adaptations to the conditions of the host and responds to feeding, in such a way that it can be modulated by prebiotics conferring health benefits, which include the increase in thermogenic capacity, in both BAT and WAT (especially subcutaneous WAT). Therefore, boosting thermogenic capacity, and therefore energy expenditure, can be added to the list of the described beneficial health effects of prebiotics. 
Moreover, the products of microbiota metabolism, so-called postbiotics, are involved in the mechanisms that connect prebiotics and microbiota modulation with thermogenesis and protection against obesity and its associated metabolic disorders. Interestingly, the direct use of postbiotics, through dietary supplementation, can also be considered as an interesting strategy to combat obesity and improve host metabolism.

In conclusion, the use of both prebiotics and postbiotics, in order to induce adaptive thermogenesis in the treatment or prevention of obesity and metabolic disorders emerges as an interesting strategy which deserves further research. Taking into account the fact that an important part of this research has been carried out in animal models, more studies are needed in humans. Moreover, the use of prebiotics represents a physiological, and even easy and affordable, way to improve health and energy metabolism. Overall, it is worthwhile to continue investigating this connection, given its therapeutic potential.

\section{REFERENCES}

Aberdein, N., Schweizer, M., and Ball, D. (2014). Sodium acetate decreases phosphorylation of hormone sensitive lipase in isoproterenol-stimulated 3T3L1 mature adipocytes. Adipocyte 3, 121-125. doi: 10.4161/adip.27936

Adam, C. L., Thomson, L. M., Williams, P. A., and Ross, A. W. (2015). Soluble fermentable dietary fibre (pectin) decreases caloric intake, adiposity and lipidaemia in high-fat diet-induced obese rats. PLOS ONE 10:e0140392. doi: 10.1371/journal.pone.0140392

Aguilar-Toalá, J. E., Garcia-Varela, R., Garcia, H. S., Mata-Haro, V., GonzálezCórdova, A. F., Vallejo-Cordoba, B., et al. (2018). Postbiotics: an evolving term within the functional foods field. Trends Food Sci. Technol. 75, 105-114. doi: 10.1016/j.tifs.2018.03.009

Alberdi, G., Rodríguez, V. M., Miranda, J., Macarulla, M. T., Churruca, I., and Portillo, M. P. (2013). Thermogenesis is involved in the bodyfat lowering effects of resveratrol in rats. Food Chem. 141, 1530-1535. doi: 10.1016/j.foodchem.2013.03.085

Althani, A. A., Marei, H. E., Hamdi, W. S., Nasrallah, G. K., El Zowalaty, M. E., Al Khodor, S., et al. (2016). Human microbiome and its association with health and diseases. J. Cell. Physiol. 231, 1688-1694. doi: 10.1002/jcp.25284

Anhê, F. F., Nachbar, R. T., Varin, T. V., Trottier, J., Dudonné, S., Le Barz, M., et al. (2018). Treatment with camu camu. Gut. doi: 10.1136/gutjnl-2017-315565. [Epub ahead of print].

Aoki, R., Kamikado, K., Suda, W., Takii, H., Mikami, Y., Suganuma, N., et al. (2017). A proliferative probiotic Bifidobacterium strain in the gut ameliorates progression of metabolic disorders via microbiota modulation and acetate elevation. Sci. Rep. 7:43522. doi: 10.1038/srep43522

Arias, N., Picó, C., Teresa Macarulla, M., Oliver, P., Miranda, J., Palou, A., et al. (2017). A combination of resveratrol and quercetin induces browning in white adipose tissue of rats fed an obesogenic diet. Obesity 25, 111-121. doi: $10.1002 /$ oby.21706

Bäckhed, F., Manchester, J. K., Semenkovich, C. F., and Gordon, J. I. (2007). Mechanisms underlying the resistance to diet-induced obesity in germ-free mice. Proc. Natl. Acad. Sci. U.S.A. 104, 979-984. doi: 10.1073/pnas.0605374104

Bartelt, A., and Heeren, J. (2014). Adipose tissue browning and metabolic health. Nat. Rev. Endocrinol. 10, 24-36. doi: 10.1038/nrendo.2013.204

Bidu, C., Escoula, Q., Bellenger, S., Spor, A., Galan, M., Geissler, A., et al. (2018). The Transplantation of $\omega 3$ PUFA-altered gut microbiota of fat- 1 mice to wildtype littermates prevents obesity and associated metabolic disorders. Diabetes 67, 1512-1523. doi: $10.2337 / \mathrm{db} 17-1488$

Boulangé, C. L., Neves, A. L., Chilloux, J., Nicholson, J. K., and Dumas, M. E. (2016). Impact of the gut microbiota on inflammation, obesity, and metabolic disease. Genome Med. 8:42. doi: 10.1186/s13073-016-0303-2

\section{AUTHOR CONTRIBUTIONS}

All authors designed the review. BR, MP, and AMR wrote the manuscript. AP and AMR revised the definitive version. All authors read and approved the final manuscript.

\section{FUNDING}

This work was supported by the Spanish Government: INTERBIOBES-AGL2015-67019-P (MINECO/FEDER, EU).

\section{ACKNOWLEDGMENTS}

CIBER de Fisiopatología de la Obesidad y Nutrición is an initiative of the Instituto de Salud Carlos III. The Laboratory of Molecular Biology, Nutrition and Biotechnology is a member of the European Network of Excellence NuGO (European Nutrigenomics Organization).

Bray, G. A., Kim, K. K., Wilding, J. P. H., and Federation, W. O. (2017). Obesity: a chronic relapsing progressive disease process. A position statement of the world obesity federation. Obes. Rev. 18, 715-723. doi: 10.1111/ob r.12551

Broeders, E. P., Nascimento, E. B., Havekes, B., Brans, B., Roumans, K. H., Tailleux, A., et al. (2015). The bile acid chenodeoxycholic acid increases human brown adipose tissue activity. Cell Metab. 22, 418-426. doi: 10.1016/j.cmet.2015. 07.002

Cani, P. D., and Delzenne, N. M. (2009). The role of the gut microbiota in energy metabolism and metabolic disease. Curr. Pharm. Des. 15, 1546-1558. doi: $10.2174 / 138161209788168164$

Cani, P. D., and Delzenne, N. M. (2010). Involvement of the gut microbiota in the development of low grade inflammation associated with obesity: focus on this neglected partner. Acta Gastroenterol. Belg. 73, 267-269.

Cani, P. D., and Everard, A. (2016). Talking microbes: when gut bacteria interact with diet and host organs. Mol. Nutr. Food Res. 60, 58-66. doi: $10.1002 / \mathrm{mnfr} .201500406$

Chakraborti, C. K. (2015). New-found link between microbiota and obesity. World J. Gastrointest. Pathophysiol. 6, 110-119. doi: 10.4291/wjgp.v6.i4.110

Chang, C. J., Lin, C. S., Lu, C. C., Martel, J., Ko, Y. F., Ojcius, D. M., et al. (2015). Ganoderma lucidum reduces obesity in mice by modulating the composition of the gut microbiota. Nat. Commun. 6:7489. doi: 10.1038/ncomms8489

Chen, M. L., Yi, L., Zhang, Y., Zhou, X., Ran, L., Yang, J., et al. (2016). Resveratrol attenuates trimethylamine-N-oxide (TMAO)-induced atherosclerosis by regulating TMAO synthesis and bile acid metabolism via remodeling of the gut microbiota. MBio 7, e02210-02215. doi: 10.1128/mBio. 02210-15

Chevalier, C., Stojanović, O., Colin, D. J., Suarez-Zamorano, N., Tarallo, V., VeyratDurebex, C., et al. (2015). Gut microbiota orchestrates energy homeostasis during cold. Cell 163, 1360-1374. doi: 10.1016/j.cell.2015.11.004

Conterno, L., Fava, F., Viola, R., and Tuohy, K. M. (2011). Obesity and the gut microbiota: does up-regulating colonic fermentation protect against obesity and metabolic disease? Genes Nutr. 6, 241-260. doi: 10.1007/s12263-011-0230-1

Crozier, A., Jaganath, I. B., and Clifford, M. N. (2009). Dietary phenolics: chemistry, bioavailability and effects on health. Nat. Prod. Rep. 26, 1001-1043. doi: 10.1039/b802662a

Davis, C. D. (2016). The gut microbiome and its role in obesity. Nutr. Today 51, 167-174. doi: 10.1097/NT.0000000000000167

De Vadder, F., Kovatcheva-Datchary, P., Goncalves, D., Vinera, J., Zitoun, C., Duchampt, A., et al. (2014). Microbiota-generated metabolites promote metabolic benefits via gut-brain neural circuits. Cell 156, 84-96. doi: $10.1016 /$ j.cell.2013.12.016 
Diamant, M., Blaak, E. E., and de Vos, W. M. (2011). Do nutrient-gut-microbiota interactions play a role in human obesity, insulin resistance and type 2 diabetes? Obes. Rev. 12, 272-281. doi: 10.1111/j.1467-789X.2010.00797.x

Dueñas, M., Muñoz-González, I., Cueva, C., Jiménez-Girón, A., Sánchez-Patán, F., Santos-Buelga, C., et al. (2015). A survey of modulation of gut microbiota by dietary polyphenols. Biomed Res. Int. 2015:850902. doi: 10.1155/2015/850902t

Etxeberria, U., Arias, N., Boque, N., Macarulla, M. T., Portillo, M. P., Martinez, J. A., et al. (2015). Reshaping faecal gut microbiota composition by the intake of trans-resveratrol and quercetin in high-fat sucrose diet-fed rats. J. Nutr. Biochem. 26, 651-660. doi: 10.1016/j.jnutbio.2015.01.002

Fak, F., Jakobsdottir, G., Kulcinskaja, E., Marungruang, N., Matziouridou, C., Nilsson, U., et al. (2015). The physico-chemical properties of dietary fibre determine metabolic responses, short-chain Fatty Acid profiles and gut microbiota composition in rats fed low- and high-fat diets. PLoS ONE 10:e0127252. doi: 10.1371/journal.pone.0127252

Gao, Z., Yin, J., Zhang, J., Ward, R. E., Martin, R. J., Lefevre, M., et al. (2009). Butyrate improves insulin sensitivity and increases energy expenditure in mice. Diabetes 58, 1509-1517. doi: 10.2337/db08-1637

Ge, H., Li, X., Weiszmann, J., Wang, P., Baribault, H., Chen, J. L., et al. (2008). Activation of $\mathrm{G}$ protein-coupled receptor 43 in adipocytes leads to inhibition of lipolysis and suppression of plasma free fatty acids. Endocrinology 149, 4519-4526. doi: 10.1210/en.2008-0059

George Kerry, R., Patra, J. K., Gouda, S., Park, Y., Shin, H. S., and Das, G. (2018). Benefaction of probiotics for human health: a review. J. Food Drug Anal. 26, 927-939. doi: 10.1016/j.jfda.2018.01.002

Geurts, L., Neyrinck, A. M., Delzenne, N. M., Knauf, C., and Cani, P. D. (2014). Gut microbiota controls adipose tissue expansion, gut barrier and glucose metabolism: novel insights into molecular targets and interventions using prebiotics. Benef. Microbes 5, 3-17. doi: 10.3920/BM2012.0065

Gibson, G. R., Hutkins, R., Sanders, M. E., Prescott, S. L., Reimer, R. A., Salminen, S. J., et al. (2017). Expert consensus document: the International Scientific Association for Probiotics and Prebiotics (ISAPP) consensus statement on the definition and scope of prebiotics. Nat. Rev. Gastroenterol. Hepatol. 14, 491-502. doi: 10.1038/nrgastro.2017.75

Hamaker, B. R., and Tuncil, Y. E. (2014). A perspective on the complexity of dietary fiber structures and their potential effect on the gut microbiota. J. Mol. Biol. 426, 3838-3850. doi: 10.1016/j.jmb.2014.07.028

Han, X., Guo, J., You, Y., Yin, M., Liang, J., Ren, C., et al. (2018). Vanillic acid activates thermogenesis in brown and white adipose tissue. Food Funct. 9, 4366-4375. doi: 10.1039/C8FO00978C

Hanatani, S., Motoshima, H., Takaki, Y., Kawasaki, S., Igata, M., Matsumura, T., et al. (2016). Acetate alters expression of genes involved in beige adipogenesis in 3T3-L1 cells and obese KK-Ay mice. J. Clin. Biochem. Nutr. 59, 207-214. doi: $10.3164 /$ jcbn.16-23

Hidalgo, M., Oruna-Concha, M. J., Kolida, S., Walton, G. E., Kallithraka, S., Spencer, J. P., et al. (2012). Metabolism of anthocyanins by human gut microflora and their influence on gut bacterial growth. J. Agric. Food Chem. 60, 3882-3890. doi: 10.1021/jf3002153

Hong, Y. H., Nishimura, Y., Hishikawa, D., Tsuzuki, H., Miyahara, H., Gotoh, C., et al. (2005). Acetate and propionate short chain fatty acids stimulate adipogenesis via GPCR43. Endocrinology 146, 5092-5099. doi: 10.1210/en.2005-0545

Hu, J., Kyrou, I., Tan, B. K., Dimitriadis, G. K., Ramanjaneya, M., Tripathi, G., et al. (2016). Short-chain fatty acid acetate stimulates adipogenesis and mitochondrial biogenesis via GPR43 in brown adipocytes. Endocrinology 157, 1881-1894. doi: 10.1210/en.2015-1944

Iebba, V., Totino, V., Gagliardi, A., Santangelo, F., Cacciotti, F., Trancassini, M., et al. (2016). Eubiosis and dysbiosis: the two sides of the microbiota. New Microbiol. 39, 1-12.

Jakobsdottir, G., Jadert, C., Holm, L., and Nyman, M. E. (2013). Propionic and butyric acids, formed in the caecum of rats fed highly fermentable dietary fibre, are reflected in portal and aortic serum. Br. J. Nutr. 110, 1565-1572. doi: 10.1017/S0007114513000809

Kalliomäki, M., Collado, M. C., Salminen, S., and Isolauri, E. (2008). Early differences in fecal microbiota composition in children may predict overweight. Am. J. Clin. Nutr. 87, 534-538. doi: 10.1093/ajcn/87.3.534

Kang, C., Wang, B., Kaliannan, K., Wang, X., Lang, H., Hui, S., et al. (2017). Gut microbiota mediates the protective effects of dietary capsaicin against chronic low-grade inflammation and associated obesity induced by high-fat diet. MBio 8:c00470-17. doi: 10.1128/mBio.00470-17

Kawamata, Y., Fujii, R., Hosoya, M., Harada, M., Yoshida, H., Miwa, M., et al. (2003). A G protein-coupled receptor responsive to bile acids. J. Biol. Chem. 278, 9435-9440. doi: 10.1074/jbc.M209706200

Keppler, K., and Humpf, H. U. (2005). Metabolism of anthocyanins and their phenolic degradation products by the intestinal microflora. Bioorg. Med. Chem. 13, 5195-5205. doi: 10.1016/j.bmc.2005.05.003

Kim, M., Furuzono, T., Yamakuni, K., Li, Y., Kim, Y. I., Takahashi, H., et al. (2017). 10-oxo-12(Z)-octadecenoic acid, a linoleic acid metabolite produced by gut lactic acid bacteria, enhances energy metabolism by activation of TRPV1. FASEB J. 31, 5036-5048. doi: 10.1096/fj.201700151R

Kimura, I., Inoue, D., Maeda, T., Hara, T., Ichimura, A., Miyauchi, S., et al. (2011). Short-chain fatty acids and ketones directly regulate sympathetic nervous system via G protein-coupled receptor 41 (GPR41). Proc. Natl. Acad. Sci. U.S.A. 108, 8030-8035. doi: 10.1073/pnas.1016088108

Kondo, T., Kishi, M., Fushimi, T., and Kaga, T. (2009). Acetic acid upregulates the expression of genes for fatty acid oxidation enzymes in liver to suppress body fat accumulation. J. Agric. Food Chem. 57, 5982-5986. doi: 10.1021/jf900470c

Labbe, S. M., Caron, A., Chechi, K., Laplante, M., Lecomte, R., and Richard, D. (2016). Metabolic activity of brown, "beige," and white adipose tissues in response to chronic adrenergic stimulation in male mice. Am. J. Physiol. Endocrinol. Metab. 311, E260-E268. doi: 10.1152/ajpendo.00545.2015

Labbe, S. M., Caron, A., Festuccia, W. T., Lecomte, R., and Richard, D. (2018). Interscapular brown adipose tissue denervation does not promote the oxidative activity of inguinal white adipose tissue in male mice. Am. J. Physiol. Endocrinol. Metab. doi: 10.1152/ajpendo.00210.2018. [Epub ahead of print].

Landsberg, L., Saville, M. E., and Young, J. B. (1984). Sympathoadrenal system and regulation of thermogenesis. Am. J. Physiol. 247(2 Pt 1), E181-E189. doi: 10.1152/ajpendo.1984.247.2.E181

Larrosa, M., Yanez-Gascon, M. J., Selma, M. V., Gonzalez-Sarrias, A., Toti, S., Ceron, J. J., et al. (2009). Effect of a low dose of dietary resveratrol on colon microbiota, inflammation and tissue damage in a DSS-induced colitis rat model. J. Agric. Food Chem. 57, 2211-2220. doi: 10.1021/jf80 $3638 \mathrm{~d}$

Lattimer, J. M., and Haub, M. D. (2010). Effects of dietary fiber and its components on metabolic health. Nutrients 2, 1266-1289. doi: 10.3390/nu2121266

Li, G., Xie, C., Lu, S., Nichols, R. G., Tian, Y., Li, L., et al. (2017). Intermittent fasting promotes white adipose browning and decreases obesity by shaping the gut microbiota. Cell Metab. 26, 672-685.e4. doi: 10.1016/j.cmet.2017. 08.019 .

Li, Z., Yi, C. X., Katiraei, S., Kooijman, S., Zhou, E., Chung, C. K., et al. (2018). Butyrate reduces appetite and activates brown adipose tissue via the gut-brain neural circuit. Gut 67, 1269-1279. doi: 10.1136/gutjnl-2017314050

Lin, H. V., Frassetto, A., Kowalik, E. J., Nawrocki, A. R., Lu, M. M., Kosinski, J. R., et al. (2012). Butyrate and propionate protect against diet-induced obesity and regulate gut hormones via free fatty acid receptor 3-independent mechanisms. PLoS ONE 7:e35240. doi: 10.1371/journal.pone.0035240

Maguire, M., and Maguire, G. (2018). Gut dysbiosis, leaky gut, and intestinal epithelial proliferation in neurological disorders: towards the development of a new therapeutic using amino acids, prebiotics, probiotics, and postbiotics. Rev. Neurosci. doi: 10.1515/revneuro-2018-0024. [Epub ahead of print].

Markowiak, P., and Slizewska, K. (2018). The role of probiotics, prebiotics and synbiotics in animal nutrition. Gut Pathog. 10:21. doi: 10.1186/s13099-018-0250-0

Marlatt, K. L., and Ravussin, E. (2017). Brown adipose tissue: an update on recent findings. Curr. Obes. Rep. 6, 389-396. doi: 10.1007/s13679-017-0283-6

Martinot, E., Sedes, L., Baptissart, M., Lobaccaro, J. M., Caira, F., Beaudoin, C., et al. (2017). Bile acids and their receptors. Mol. Aspects Med. 56, 2-9. doi: 10.1016/j.mam.2017.01.006

Maruyama, T., Miyamoto, Y., Nakamura, T., Tamai, Y., Okada, H., Sugiyama, E., et al. (2002). Identification of membrane-type receptor for bile acids (M-BAR). Biochem. Biophys. Res. Commun. 298, 714-719. doi: 10.1016/S0006-291X(02)02550-0

Mestdagh, R., Dumas, M. E., Rezzi, S., Kochhar, S., Holmes, E., Claus, S. P., et al. (2012). Gut microbiota modulate the metabolism of brown adipose tissue in mice. J. Proteome Res. 11, 620-630. doi: 10.1021/pr200938v 
Meydani, M., and Hasan, S. T. (2010). Dietary polyphenols and obesity. Nutrients 2, 737-751. doi: 10.3390/nu2070737

Milani, C., Duranti, S., Bottacini, F., Casey, E., Turroni, F., Mahony, J., et al. (2017). The first microbial colonizers of the human gut: composition, activities, and health implications of the infant gut microbiota. Microbiol. Mol. Biol. Rev. 81, e00036-17. doi: 10.1128/MMBR.00036-17

Moreno-Navarrete, J. M., Serino, M., Blasco-Baque, V., Azalbert, V., Barton, R. H., Cardellini, M., et al. (2018). Gut microbiota interacts with markers of adipose tissue browning, insulin action and plasma acetate in morbid obesity. Mol. Nutr. Food Res. 62:1700721. doi: 10.1002/mnfr.201700721

Mosqueda-Solis, A., Sanchez, J., Portillo, M. P., Palou, A., and Pico, C. (2018). Combination of capsaicin and hesperidin reduces the effectiveness of each compound to decrease the adipocyte size and to induce browning features in adipose tissue of western diet fed rats. J. Agric. Food Chem. 66, 9679-9689. doi: 10.1021 /acs.jafc. 8 b02611

Murakami, Y., Ojima-Kato, T., Saburi, W., Mori, H., Matsui, H., Tanabe, S., et al. (2015). Supplemental epilactose prevents metabolic disorders through uncoupling protein-1 induction in the skeletal muscle of mice fed high-fat diets. Br. J. Nutr. 114, 1774-1783. doi: 10.1017/S00071145150 03505

Nedergaard, J., Bengtsson, T., and Cannon, B. (2007). Unexpected evidence for active brown adipose tissue in adult humans. Am. J. Physiol. Endocrinol. Metab. 293, E444-E452. doi: 10.1152/ajpendo.00691.2006

Nedergaard, J., and Cannon, B. (2014). The browning of white adipose tissue: some burning issues. Cell Metab. 20, 396-407. doi: 10.1016/j.cmet.2014. 07.005

Neyrinck, A. M., Bindels, L. B., Geurts, L., Van Hul, M., Cani, P. D., and Delzenne, N. M. (2017). A polyphenolic extract from green tea leaves activates fat browning in high-fat-diet-induced obese mice. J. Nutr. Biochem. 49, 15-21. doi: 10.1016/j.jnutbio.2017.07.008

Palou, A., Picó, C., Bonet, M. L., and Oliver, P. (1998). The uncoupling protein, thermogenin. Int. J. Biochem. Cell Biol. 30, 7-11. doi: 10.1016/S1357-2725(97)00065-4

Palou, M., Sánchez, J., García-Carrizo, F., Palou, A., and Picó, C. (2015). Pectin supplementation in rats mitigates age-related impairment in insulin and leptin sensitivity independently of reducing food intake. Mol. Nutr. Food Res. 59, 2022-2033. doi: 10.1002/mnfr.201500292

Pascale, A., Marchesi, N., Marelli, C., Coppola, A., Luzi, L., Govoni, S., et al. (2018). Microbiota and metabolic diseases. Endocrine 61, 357-371. doi: 10.1007/s12020-018-1605-5

Perry, R. J., Peng, L., Barry, N. A., Cline, G. W., Zhang, D., Cardone, R. L., et al. (2016). Acetate mediates a microbiome-brain-beta-cell axis to promote metabolic syndrome. Nature 534, 213-217. doi: 10.1038/natur e18309

Petschow, B., Dore, J., Hibberd, P., Dinan, T., Reid, G., Blaser, M., et al. (2013). Probiotics, prebiotics, and the host microbiome: the science of translation. Ann. N.Y. Acad. Sci. 1306, 1-17. doi: 10.1111/nyas. 12303

Pico, C., and Palou, A. (2013). Perinatal programming of obesity: an introduction to the topic. Front. Physiol. 4:255. doi: 10.3389/fphys.2013.00255

Requena, T., Martinez-Cuesta, M. C., and Pelaez, C. (2018). Diet and microbiota linked in health and disease. Food Funct. 9, 688-704. doi: 10.1039/C7FO0 $1820 \mathrm{G}$

Roberfroid, M., Gibson, G. R., Hoyles, L., McCartney, A. L., Rastall, R., Rowland, I., et al. (2010). Prebiotic effects: metabolic and health benefits. Br. J. Nutr. 104(Suppl. 2), S1-S63. doi: 10.1017/S0007114510003363

Rodriguez, A. M., and Palou, A. (2004). Uncoupling proteins: gender-dependence and their relation to body weight control. Int. J. Obes. Relat. Metab. Disord. 28, 327-329. doi: 10.1038/sj.ijo.0802579

Rothwell, N. J., and Stock, M. J. (1979). A role for brown adipose tissue in diet-induced thermogenesis. Nature 281, 31-35. doi: 10.1038/28 $1031 \mathrm{a} 0$

Sahuri-Arisoylu, M., Brody, L. P., Parkinson, J. R., Parkes, H., Navaratnam, N., Miller, A. D., et al. (2016). Reprogramming of hepatic fat accumulation and "browning" of adipose tissue by the short-chain fatty acid acetate. Int. J. Obes. 40, 955-963. doi: 10.1038/ijo.2016.23

Sakakibara, S., Yamauchi, T., Oshima, Y., Tsukamoto, Y., and Kadowaki, T. (2006). Acetic acid activates hepatic AMPK and reduces hyperglycemia in diabetic KK-A(y) mice. Biochem. Biophys. Res. Commun. 344, 597-604. doi: 10.1016/j.bbrc.2006.03.176

Sanchez, D., Muguerza, B., Moulay, L., Hernandez, R., Miguel, M., and Aleixandre, A. (2008). Highly methoxylated pectin improves insulin resistance and other cardiometabolic risk factors in Zucker fatty rats. J. Agric. Food Chem. 56, 3574-3581. doi: 10.1021/jf703598j

Schonfeld, P., and Wojtczak, L. (2016). Short- and medium-chain fatty acids in energy metabolism: the cellular perspective. J. Lipid Res. 57, 943-954. doi: 10.1194/jlr.R067629

Serrano, A., Asnani-Kishnani, M., Rodriguez, A. M., Palou, A., Ribot, J., and Bonet, M. L. (2018). Programming of the beige phenotype in white adipose tissue of adult mice by mild resveratrol and nicotinamide riboside supplementations in early postnatal life. Mol. Nutr. Food Res. 62:e1800463. doi: $10.1002 / \mathrm{mnfr} .201800463$

Slavin, J. (2013). Fiber and prebiotics: mechanisms and health benefits. Nutrients 5, 1417-1435. doi: 10.3390/nu5041417

Somm, E., Henry, H., Bruce, S. J., Aeby, S., Rosikiewicz, M., Sykiotis, G. P., et al. (2017). $\beta$-Klotho deficiency protects against obesity through a crosstalk between liver, microbiota, and brown adipose tissue. JCI Insight 2:91809. doi: $10.1172 /$ jci.insight.91809

Suárez-Zamorano, N., Fabbiano, S., Chevalier, C., Stojanović, O., Colin, D. J., Stevanović, A., et al. (2015). Microbiota depletion promotes browning of white adipose tissue and reduces obesity. Nat. Med. 21, 1497-1501. doi: 10.1038/nm.3994

Teodoro, J. S., Zouhar, P., Flachs, P., Bardova, K., Janovska, P., Gomes, A. P., et al. (2014). Enhancement of brown fat thermogenesis using chenodeoxycholic acid in mice. Int. J. Obes. 38, 1027-1034. doi: 10.1038/ijo.20 13.230

Topping, D. L., and Clifton, P. M. (2001). Short-chain fatty acids and human colonic function: roles of resistant starch and nonstarch polysaccharides. Physiol. Rev. 81, 1031-1064. doi: 10.1152/physrev.2001.81. 3.1031

Trent, C. M., and Blaser, M. J. (2016). Microbially produced acetate: a "missing link" in understanding obesity? Cell Metab. 24, 9-10. doi: 10.1016/j.cmet.2016.06.023

Villanueva-Millan, M. J., Perez-Matute, P., and Oteo, J. A. (2015). Gut microbiota: a key player in health and disease. A review focused on obesity. J. Physiol. Biochem. 71, 509-525. doi: 10.1007/s13105-0150390-3

Wang, S., Liang, X., Yang, Q., Fu, X., Zhu, M., Rodgers, B. D., et al. (2017). Resveratrol enhances brown adipocyte formation and function by activating AMP-activated protein kinase (AMPK) $\alpha 1$ in mice fed high-fat diet. Mol. Nutr. Food Res. 61. doi: 10.1002/mnfr.2016 00746

Wang, S., Zhu, M. J., and Du, M. (2015). Prevention of obesity by dietary resveratrol: how strong is the evidence? Expert Rev. Endocrinol. Metab. 10, 561-564. doi: 10.1586/17446651.2015.1096771

Watanabe, M., Houten, S. M., Mataki, C., Christoffolete, M. A., Kim, B. W., Sato, H., et al. (2006). Bile acids induce energy expenditure by promoting intracellular thyroid hormone activation. Nature 439, 484-489. doi: 10.1038/nature04330

Weitkunat, K., Stuhlmann, C., Postel, A., Rumberger, S., Fankhänel, M., Woting, A., et al. (2017). Short-chain fatty acids and inulin, but not guar gum, prevent diet-induced obesity and insulin resistance through differential mechanisms in mice. Sci. Rep. 7:6109. doi: 10.1038/s41598-017-0 $6447-\mathrm{x}$

Wu, J., Boström, P., Sparks, L. M., Ye, L., Choi, J. H., Giang, A. H., et al. (2012). Beige adipocytes are a distinct type of thermogenic fat cell in mouse and human. Cell 150, 366-376. doi: 10.1016/j.cell.2012.05.016

You, Y., Han, X., Guo, J., Guo, Y., Yin, M., Liu, G., et al. (2018). Cyanidin3-glucoside attenuates high-fat and high-fructose diet-induced obesity by promoting the thermogenic capacity of brown adipose tissue. J. Funct. Foods 41:10. doi: 10.1016/j.jff.2017.12.025

You, Y., Yuan, X., Lee, H. J., Huang, W., Jin, W., and Zhan, J. (2015). Mulberry and mulberry wine extract increase the number of mitochondria during brown adipogenesis. Food Funct. 6, 401-408. doi: 10.1039/C4FO0 0719K 
You, Y., Yuan, X., Liu, X., Liang, C., Meng, M., Huang, Y., et al. (2017). Cyanidin-3-glucoside increases whole body energy metabolism by upregulating brown adipose tissue mitochondrial function. Mol. Nutr. Food Res. 61. doi: 10.1002/mnfr.201700261

Zhang, Y. J., Li, S., Gan, R. Y., Zhou, T., Xu, D. P., and Li, H. B. (2015). Impacts of gut bacteria on human health and diseases. Int. J. Mol. Sci. 16, 7493-7519. doi: $10.3390 /$ ijms 16047493

Zietak, M., Chabowska-Kita, A., and Kozak, L. P. (2017). Brown fat thermogenesis: stability of developmental programming and transient effects of temperature and gut microbiota in adults. Biochimie 134, 93-98. doi: 10.1016/j.biochi.2016.12.006

Zietak, M., Kovatcheva-Datchary, P., Markiewicz, L. H., Ståhlman, M., Kozak, L. P., and Bäckhed, F. (2016). Altered microbiota contributes to reduced diet-induced obesity upon cold exposure. Cell Metab. 23, 1216-1223. doi: 10.1016/j.cmet.2016. 05.001
Zietak, M., and Kozak, L. P. (2016). Bile acids induce uncoupling protein 1-dependent thermogenesis and stimulate energy expenditure at thermoneutrality in mice. Am. J. Physiol. Endocrinol. Metab. 310, E346-E354. doi: 10.1152/ajpendo.00485.2015

Conflict of Interest Statement: The authors declare that the research was conducted in the absence of any commercial or financial relationships that could be construed as a potential conflict of interest.

Copyright $\odot 2019$ Reynés, Palou, Rodríguez and Palou. This is an open-access article distributed under the terms of the Creative Commons Attribution License (CC BY).

The use, distribution or reproduction in other forums is permitted, provided the original author(s) and the copyright owner(s) are credited and that the original publication in this journal is cited, in accordance with accepted academic practice. No use, distribution or reproduction is permitted which does not comply with these terms. 